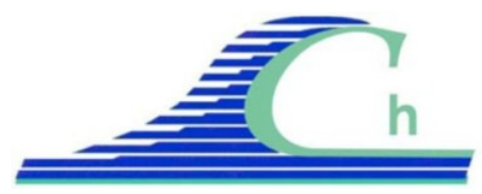

XII ${ }^{\text {èmes }}$ Journées Nationales Génie Côtier - Génie Civil

Cherbourg, $12-14$ juin 2012

DOI:10.5150/jngcgc.2012.088-B C Editions Paralia CFL

disponible en ligne - http://www.paralia.fr - available online

\title{
Impact hydrodynamique de la mise en place d'un parc d'hydroliennes en Baie du Mont Saint-Michel
}

\author{
François BATIFOULIER ${ }^{1}$, Youen KERVELLA ${ }^{1}$, Renaud LABORBE ${ }^{1}$, \\ Jérôme CUNY ${ }^{1}$, Matthieu CAILLAUD ${ }^{1,2}$, \\ Pascal LAZURE ${ }^{3}$, Florence CAYOCCA ${ }^{3}$
}

\author{
1. OPEN OCEAN, Pépinière Créatic, 115 rue Claude CHAPPE, Technopole Brest \\ Iroise, Site de la Pointe du Diable, 29280 Plouzané, France. www.openocean.fr \\ francois.batifoulier@openocean.fr \\ 2. Capital Sécurité Environnement, 16 rue Gallieni, BP 12377 - 98802 Nouméa Cedex, \\ Nouvelle Calédonie. \\ 3. IFREMER, DYNECO/PHYSED, Centre de Brest, 29280 Plouzané, France.
}

\section{Résumé :}

Les fortes vitesses rencontrées à l'entrée de la Baie du Mont Saint-Michel font de cet environnement macro-tidal un endroit privilégié, en termes de ressources énergétiques, pour l'implantation d'une ferme d'hydroliennes. Afin d'établir plus précisément son potentiel énergétique et d'évaluer l'impact de l'implantation de telles machines sur son hydrodynamisme, une modélisation hydrodynamique de la Baie du Mont Saint-Michel est mise en œuvre à l'aide du modèle de circulation régionale MARS3D (LAZURE \& DUMAS, 2008) dans sa version tridimensionnelle.

La validation hydrodynamique de cette configuration est réalisée à partir de comparaisons à des mesures courantologiques récentes (KERVELLA, 2010). A partir de cette modélisation, une cartographie du potentiel énergétique de la Baie est établie et les endroits propices à l'installation de structures récupératrices de l'énergie des courants sont identifiés.

L'impact de l'implantation d'une ou plusieurs structures à l'un de ces endroits sur l'hydrodynamique de la Baie est évalué : l'effet de l'extraction d'énergie des courants est modélisé par l'implémentation d'un nouveau terme puits, correspondant à une force de retard colinéaire à l'écoulement (DEFNE et al., 2011), dans les équations du mouvement de MARS3D. Les résultats présentés pour 2 valeurs d'extraction de l'énergie disponible (25\% et 55\%) montrent que l'impact d'un parc de petite superficie sur les courants reste très local.

Mots-clés : Modélisation régionale - Baie du Mont Saint-Michel - Hydrodynamisme Potentiel énergétique - Hydroliennes

\section{Introduction}

A l'heure où plusieurs permis de construire pour des projets éoliens en bordure de la Baie du Mont Saint-Michel viennent d'être refusés en raison du classement du Mont 
Saint-Michel au patrimoine mondial de l'UNESCO, la question se pose quant à l'utilisation des forts courants de marée de la Baie (fortes vitesses rencontrées à l'entrée de la Baie (EHROLD, 1999 ; CAYOCCA et al., 2006). En effet, récupérer cette ressource énergétique régulière et prévisible ne dénaturerait en rien le paysage visible depuis le Mont et ses alentours.

L'installation de telles machines peut cependant avoir des conséquences sur les écosystèmes et les cultures de la Baie, notamment de par les modifications hydrodynamiques qu'elles entraînent. En fonction de la superficie des parcs installés, la circulation au sein de la Baie peut être modifiée et des zones d'envasement pourraient apparaître.

Dans ce contexte, un modèle numérique a été implanté sur le domaine de la Baie du Mont Saint-Michel afin d'évaluer en première approche l'impact de l'installation d'un parc d'hydroliennes sur les courants. Après une brève description du modèle et de la façon dont le parc est pris en compte dans les équations du mouvement, cet article présente successivement la validation du modèle hydrodynamique par le biais de mesures in-situ, le potentiel énergétique de la Baie et le choix d'une zone d'implantation pour le parc hydrolien, et enfin l'évaluation de l'impact d'un parc de petite superficie sur les courants de la Baie.

\section{Description du modèle et prise en compte du parc d'hydroliennes}

\subsection{Le modèle hydrodynamique MARS3D}

Le modèle hydrodynamique MARS3D (LAZURE \& DUMAS, 2008) est ici utilisé dans sa version tridimensionnelle avec 10 niveaux sur la verticale (coordonnées sigma). Le maillage cartésien, dont la résolution spatiale est de $200 \mathrm{~m}$ par $200 \mathrm{~m}$, couvre toute la Baie du Mont Saint-Michel et s'étend au nord jusqu'à Barneville-Carteret (49²3' N). La bathymétrie (figure 1) a été construite à l'aide du logiciel BMGTOOLS (Ifremer) à partir de modèles numériques de terrain au pas de $100 \mathrm{~m}$ réalisés à partir de levés LIDAR et par sondeur mono-faisceau (IFREMER). Les conditions aux limites ouvertes (nord et ouest) sont fournies en hauteur d'eau, et calculées à partir de 115 harmoniques de marée (LE ROY \& SIMON, 2003). Le niveau moyen est également prescrit sur l'ensemble de la zone d'étude. Au niveau des forçages atmosphériques, les sorties du modèle ARPEGE de Météo-France (15 km de résolution) sont utilisées. Le calage hydrodynamique du modèle se fait par le biais de dissipation d'énergie par frottement sur le fond. Des zones de frottements sont définies: longueur de rugosité macroscopique $\left(\mathrm{z}_{0}\right)$ de 0,1 $\mathrm{mm}$ dans l'ensemble de la Baie et de 0,25 $\mathrm{mm}$ au nord de la Baie ; une zone de frottements plus importants $\left(\mathrm{z}_{0}=10 \mathrm{~mm}\right)$ a été définie à l'ouest de la Baie (au sud de Cancale) afin de représenter l'effet cumulé des nombreuses tables à huîtres et des banquettes vaseuses à cet endroit (à l’instar de KERVELLA, 2010). 


\section{XII ${ }^{\text {èmes }}$ Journées Nationales Génie Côtier - Génie Civil \\ Cherbourg, 12-14 juin 2012}

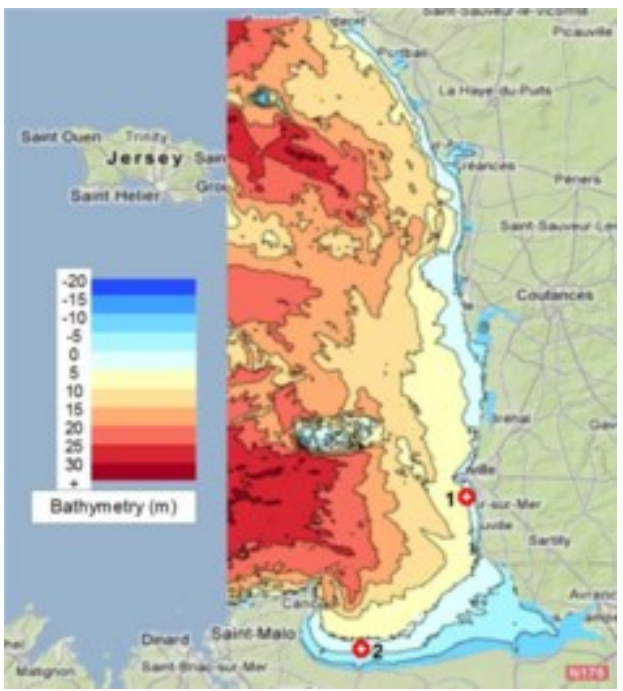

Figure 1. Bathymétrie de la Baie du Mont Saint-Michel. Position des points de mesures des campagnes EPEL (point 1) et VBM (point 2).

\subsection{Prise en compte du parc d'hydroliennes}

Afin d'évaluer l'impact que peut avoir un parc d'hydroliennes sur les courants, à proximité ou même en champ lointain, une première formulation est mise en place dans les équations du mouvement de MARS3D. Pour calibrer cette formulation, le parc est assimilé à une maille du domaine (200 m × $200 \mathrm{~m}$ ) sur l'horizontale et a une hauteur de 15 m, soit avec toutes les simplifications que cela entraîne, un "petit" parc de 6 ou 7 machines dont le diamètre des rotors serait d'environ $12 \mathrm{~m}$. En effet, les espacements entre machines sont généralement de l'ordre de 5 à 10 fois le diamètre des rotors. Un diamètre de rotor de $12 \mathrm{~m}$ est au jour d'aujourd'hui une valeur moyenne si on considère de récentes implantations comme les 2 turbines de Marine Current Turbines Ltd., l'une Seaflow, d'un diamètre de $11 \mathrm{~m}$ installée au large de l'Angleterre et l'autre, Seagen, d'un diamètre de $16 \mathrm{~m}$ installée au Nord de l'Irlande, ainsi que l'hydrolienne OpenHydro de $16 \mathrm{~m}$ récemment déployée à Paimpol-Bréhat.

Dans la formulation utilisée, l'effet de l'extraction d'énergie des courants est modélisé par l'implémentation d'un nouveau terme puits, correspondant à une force de retard $F$ colinéaire à l'écoulement (DEFNE et al., 2011 ; AHMADIAN et al., 2012) :

$\vec{F}=-\frac{B_{i m}}{|\vec{F}|} \cdot \frac{\vec{F}}{|\vec{v}|}$

Avec $V$ vecteur vitesse au point considéré et $P_{\text {ext }}$ la puissance extraite du courant par le parc. Cette puissance extraite $P_{\text {ext }}$ est fonction de la puissance disponible dans la cellule au temps d'extraction et d'un taux d'extraction, généralement compris entre 0,35 et 0,5 pour une turbine (MYERS \& BAHAJ, 2006), dépendant de l'efficacité de cette turbine. L'effet d'interaction entre hydroliennes est encore assez méconnu malgré quelques études récentes (DIVETT et al., 2011 ; MYCEK et al., 2011). Dans cette étude, nous examinons donc l'effet d'un parc en nous basant sur 2 taux d'extraction d'énergie 
arbitraires, 0,25 (scénario avec extraction modérée) et 0,55 (scénario avec extraction importante), et une orientation d'hydrolienne vis-à-vis des courants fixes.

Les termes ajoutés aux équations sont donc :

$$
\begin{aligned}
& F_{\mathrm{u}}=-C_{\mathrm{ex} *} \frac{1}{2} \cdot \rho_{\mathrm{n}} u_{\mathrm{r}} \sin (\theta) \cdot|\overrightarrow{\mathrm{V}}| \\
& F_{v}=-C_{e x t} \frac{1}{2}, \rho_{n} v_{n} \cos (\theta),|\vec{V}|
\end{aligned}
$$

Avec $\theta$ angle entre l'axe de rotation des hydroliennes et l'axe y (sud-nord) de notre maillage et $C_{\text {ext }}$ un coefficient qui permet d'obtenir l'extraction de puissance désirée, ajusté après plusieurs simulations successives.

Une fois le coefficient $C_{\text {ext }}$ calibré sur une maille, les forces de retard seront ajoutées dans 10 mailles (soit un parc d'une superficie de $0,4 \mathrm{~km}^{2}$ avec environ une cinquantaine de machines) et sur les couches verticales concernées.

\section{Résultats}

\subsection{Comparaisons aux mesures in-situ}

Deux jeux de données ont été utilisés afin de calibrer le modèle : les mesures de la campagne EPEL de février 2003 et celles de la campagne VMB de février-mars 2009. Les données EPEL ont été acquises avec une bouée S4 au point $\left(1^{\circ} 40^{\prime} \mathrm{W} ; 48^{\circ} 49^{\prime} \mathrm{N}\right.$, cf figure 1 point 1$)$ et les données VBM avec un ADCP au point $\left(1^{\circ} 47^{\prime} \mathrm{W} ; 48^{\circ} 38^{\prime} \mathrm{N}\right.$, cf figure 1 point 2).

La comparaison des mesures EPEL et des résultats du modèle montre que la vitesse zonale est généralement bien représentée (cf figure 2, en haut), elle est cependant surestimée pour les vitesses fortes au jusant (vitesses zonales négatives), l'erreur RMS est de $11 \mathrm{~cm} \mathrm{~s}^{-1}$. La vitesse méridienne (cf figure 2, en bas), plus faible que la vitesse zonale, est moins bien représentée. Elle est généralement sous-estimée en flot (vitesses zonales positives) et surestimée en jusant (vitesses zonales négatives), l'erreur RMS est de $18 \mathrm{~cm} \mathrm{~s}^{-1}$. La vitesse méridienne est plus difficile à obtenir puisqu'elle est plus liée à une circulation locale résultant de la bathymétrie (lissée dans notre modèle avec des mailles à 200 m) qu'à la propagation directe de la marée.

Les mesures VBM (cf figure 3) sont globalement bien représentées tant pour la vitesse zonale que méridienne à part le 23 et le 24 février 2009 où les mesures pourraient être influencées par un vent local mal représenté dans notre forçage de vent avec $15 \mathrm{~km}$ de résolution. Les erreurs RMS sont de $12 \mathrm{~cm} \mathrm{~s}^{-1}$ pour la vitesse zonale et de $13 \mathrm{~cm} \mathrm{~s}^{-1}$ pour la vitesse méridienne. Les jeux de mesures EPEL et VBM ont permis de calibrer le modèle, qui globalement, reproduit de manière satisfaisante ces observations.

\subsection{Potentiel énergétique de la Baie}

L'analyse de l'énergie annuelle moyenne (simulation d'un an sur 2003, figure 4) montre qu'en termes de courants, le potentiel énergétique de la Baie est essentiellement 


\section{XII ${ }^{\text {èmes }}$ Journées Nationales Génie Côtier - Génie Civil \\ Cherbourg, 12-14 juin 2012}

concentré autour de la pointe du Grouin avec des valeurs moyennes locales de plus de $400 \mathrm{~W} / \mathrm{m}^{2}$. Le reste de la Baie présente des valeurs moyennes faibles à l'exception de la partie Est de la Baie où les résultats sont plus discutables du fait de la très forte dynamique de la morphologie des fonds sableux (formation de chenaux, CAYOCCA et al., 2006), non prise en compte dans notre modèle.

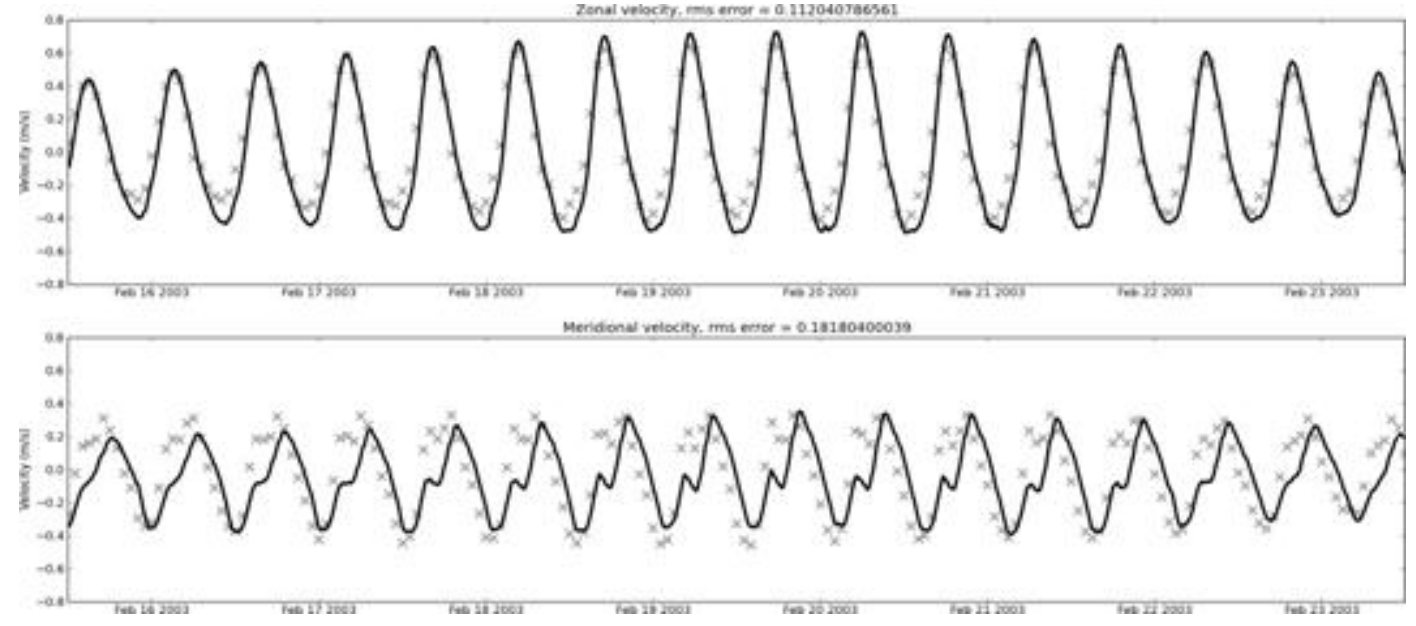

Figure 2. Comparaison des mesures EPEL (croix grises) et des résultats du modèle (trait noir), en haut pour la vitesse zonale et en bas pour la vitesse méridienne.

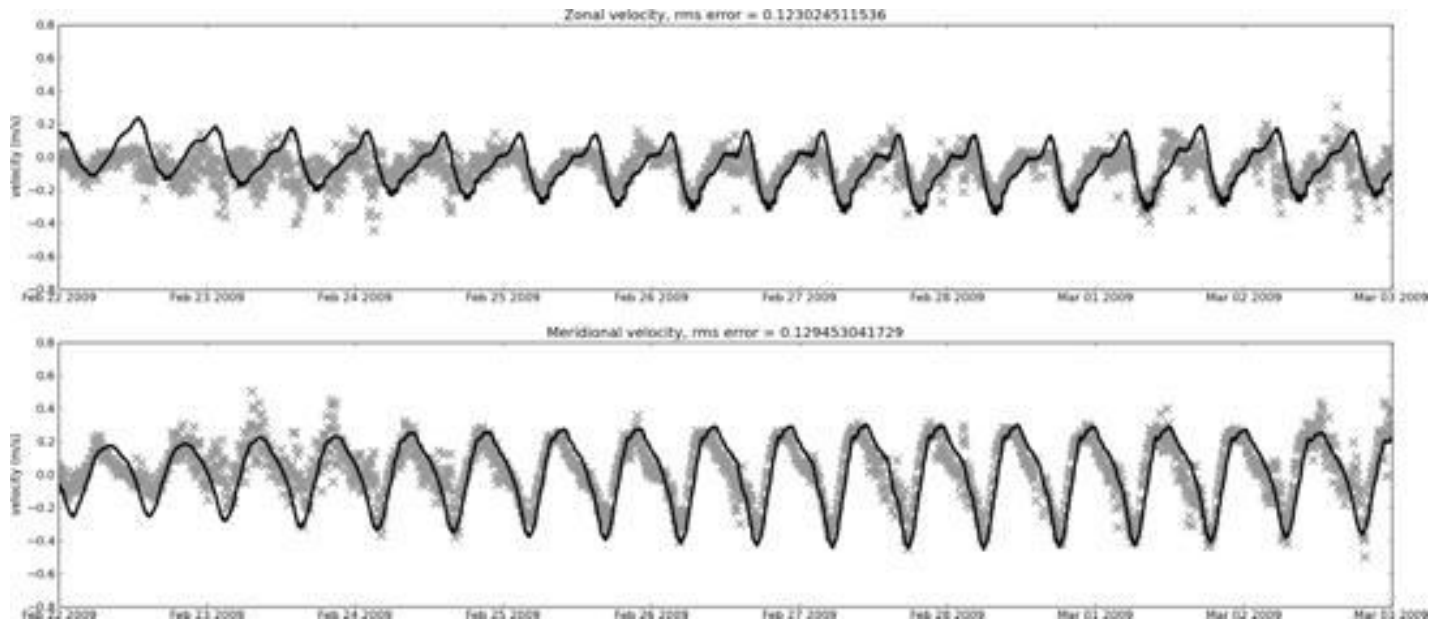

Figure 3. Comparaison des mesures VBM (croix grises) et des résultats du modèle (trait noir), en haut pour la vitesse zonale et en bas pour la vitesse méridienne.

La zone au large de la pointe du Grouin est donc propice à l'implantation de notre parc d'hydroliennes. Néanmoins, il convient aussi d'examiner d'autres paramètres essentiels à l'implantation d'un parc qui sont, outre les aspects socio-économiques non-abordés dans cette étude, la hauteur d'eau sur le site au cours du temps et la distance à la côte pour un futur raccordement électrique. La hauteur d'eau est très variable au cours du temps dans cet environnement macrotidal (plus de $12 \mathrm{~m}$ de marnage). 


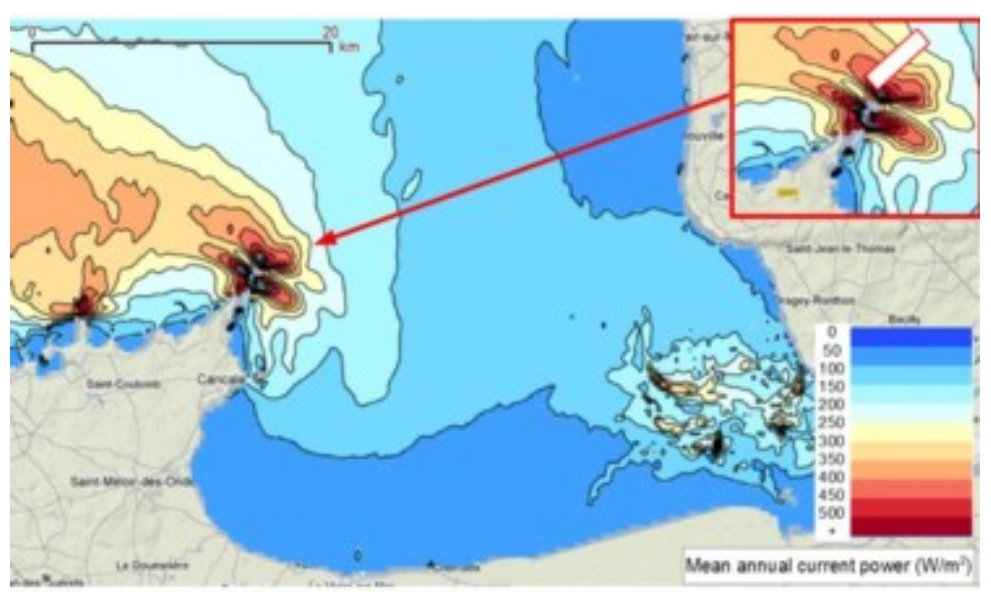

Figure 4. Puissance moyenne annuelle de la Baie du Mont Saint-Michel calculée sur l'année 2003. Le rectangle blanc en haut à droite représente la zone où est implanté dans le modèle le parc d'hydroliennes.

La zone retenue $\left(1,83^{\circ} \mathrm{W}\right.$ à $1,82^{\circ} \mathrm{W}, 48,73^{\circ} \mathrm{N}$ à $\left.48,74^{\circ} \mathrm{N}\right)$ est située à $2 \mathrm{~km}$ de la côte et présente une côte bathymétrique moyenne de $25 \mathrm{~m}$ par rapport au niveau moyen de la mer. La valeur de la puissance moyenne annuelle des courants y est d'environ $400 \mathrm{~W} / \mathrm{m}^{2}$. L'énergie est d'abord extraite sur une seule maille afin de calibrer la valeur de $C_{e x t}$ puis sur 10 mailles sur la zone définie (figure 4). Afin d'extraire un maximum de l'énergie disponible, les hydroliennes sont positionnées orthogonalement à la direction des courants principaux, soit à $\theta=70^{\circ}$ par rapport au Nord.

\subsection{Calibrage de l'extraction sur une maille}

Des simulations successifs ont permis d'ajuster la valeur du coefficient $C_{\text {ext }}$ afin d'obtenir l'extraction de l'énergie désirée au point de la maille de l'hydrolienne. Deux valeurs d'extraction ont été choisies, un taux d'extraction de 0,25 $\left(C_{e x t}=2 \times 10^{-3}\right)$ et un taux de $0,55\left(C_{\text {ext }}=8 \times 10^{-3}\right)$.

La figure 5 présente les modifications des profils verticaux de vitesse sur la maille où a lieu l'extraction d'énergie à deux instants, au pic de jusant (cf figure 5 , en haut) et au pic de flot (cf figure 5, en flot). Ces profils montrent, que ce soit à marée montante ou descendante, une atténuation des vitesses zonales et méridiennes en valeur absolue dans les quinze premiers mètres de la colonne d'eau où est appliquée la force de retard, par rapport à une simulation de référence sans hydrolienne. L'atténuation est sensiblement plus forte pour le taux d'extraction de 0,55. En revanche, en surface, la vitesse zonale est légèrement plus forte dans les cas avec hydroliennes. L'ajout d'hydroliennes au fond engendre donc une accélération des courants en surface.

Les valeurs du $C_{\text {ext }}$ étant maintenant calibrées, l'extraction est appliquée sur les 10 mailles définies précédemment (cf figure 2). 

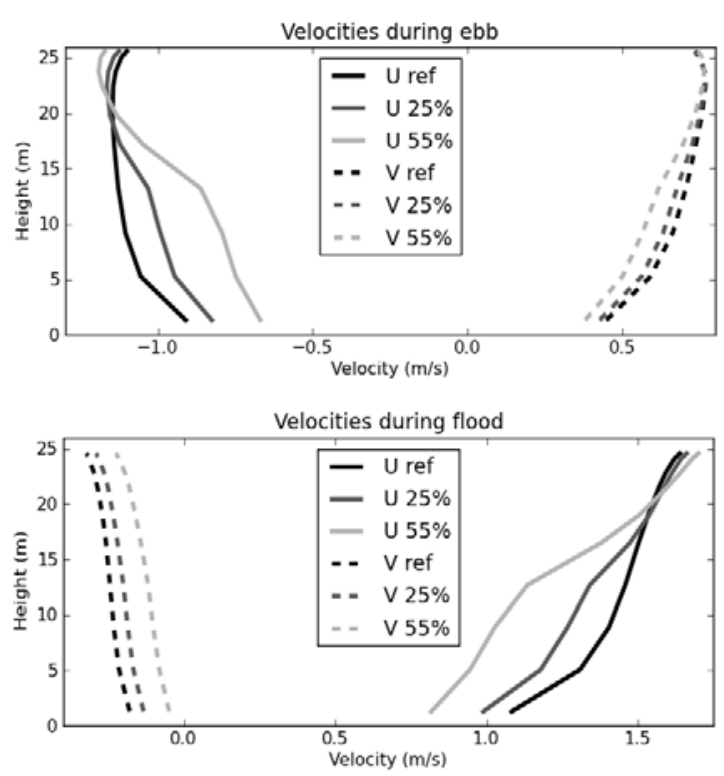

Figure 5. Profils verticaux des vitesses zonales (U) et méridiennes (V) en jusant (en haut) et en flot (en bas) pour une simulation de référence et deux simulations avec des taux d'extraction de 0,25 et de 0,55, au niveau de la maille où l'extraction est implémentée.

\subsection{Impact d'un parc d'hydroliennes}

La figure 6 présente la distribution de la différence des moyennes annuelles de vitesses (moyennées sur la verticale) entre une simulation de référence sans hydrolienne et une simulation avec un parc (constitué de 10 mailles) qui extrait $25 \%$ de la puissance des courants disponible (en haut) et une simulation avec un parc qui extrait 55\% de la puissance des courants disponible (en bas). Localement, cette différence peut être supérieure à $10 \mathrm{~cm} \mathrm{~s}^{-1}$ dans le cas où le taux d'extraction est de 55\%. Cette moyenne annuelle semble montrer que seuls les abords du parc sont affectés (dans des mesures différentes selon le taux d'extraction) mais que l'impact sur le reste de la baie est limité. Rappelons que la superficie de notre parc est de $0,4 \mathrm{~km}^{2}$ (environ 50 machines) alors que dans de récentes études, 2000 machines de $10 \mathrm{~m}$ de diamètre étaient incorporées dans les simulations (AHMADIAN et al., 2012) et qu'en réalité, la taille des futurs parcs hydroliens pourrait être de plusieurs $\mathrm{km}^{2}$.

Il est néanmoins intéressant d'évaluer l'impact du parc en d'autres points de la baie plus éloignés : le point "Cancale" près des installations ostréicoles de Cancale (cf figure 6, point 1), le point "Vivier" (cf figure 6, point 2) près des installations ostréicoles et mytilicoles du Vivier-sur-Mer et le point "MSM" à l'Est de la baie à proximité du Mont Saint-Michel (cf figure 6, point 3). Ces 3 points représentent des zones d'intérêt pour les usagers de la baie du fait de leur tendance à un envasement qui peut être très néfaste pour leurs activités respectives. 


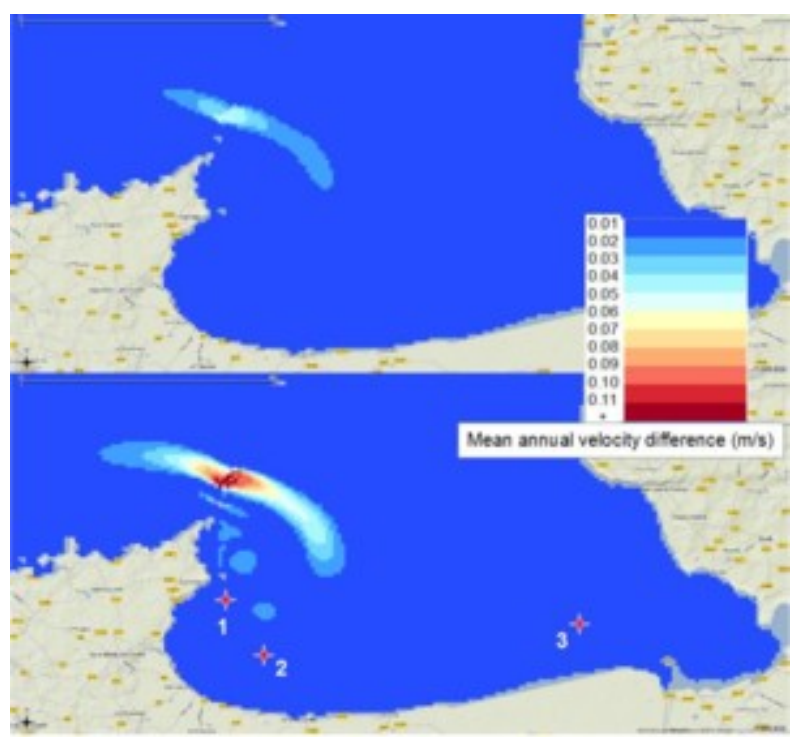

Figure 6. Différence des vitesses barotropes moyennées sur l'année 2003 entre une simulation de référence sans hydrolienne et une simulation avec un parc de 10 mailles qui extrait 25\% de la puissance des courants disponible (en haut) et une simulation avec un parc qui extrait 55\% de la puissance des courants disponible (en bas).

La figure 7 (haut) présente les niveaux de la mer (SSH) aux trois différents points pour la simulation de référence et la simulation avec une extraction de 55\%. Elle montre que les différentes courbes sont confondues, le signal de marée en niveau arrive donc simultanément aux différents points et l'ajout des hydroliennes modifie peu la propagation de la marée. Les différences de niveaux aux trois points entre la simulation de référence et la simulation avec une extraction de 55\% sont en effet inférieures à $1 \mathrm{~cm}$ (cf figure 7, en bas).

Au niveau de l'intensité du courant, les résultats entre la simulation de référence et la simulation avec une extraction de 55\% montrent localement quelques différences (cf figure 8, en haut). Les différences d'intensité entre les deux simulations montrent que les différences sont faibles pour le point MSM ( $<1 \mathrm{~cm} \mathrm{~s}^{-1}$ ) (cf figure 8, en bas). Pour les points Vivier et Cancale les différences d'intensité du courant sont plus importantes et peuvent atteindre $3 \mathrm{~cm} \mathrm{~s}^{-1}$ et $5 \mathrm{~cm} \mathrm{~s}^{-1}$ respectivement.

\section{Conclusions et perspectives}

Le potentiel énergétique annuel (2003) moyen de la Baie du Mont Saint-Michel est présenté dans cet article. La zone d'intérêt pour l'implantation d'un parc d'hydroliennes se situe essentiellement autour de la pointe du Grouin (entrée de la Baie, au nord de Cancale), la bathymétrie y étant de surcroît favorable. 


\section{XII ${ }^{\text {èmes }}$ Journées Nationales Génie Côtier - Génie Civil \\ Cherbourg, 12-14 juin 2012}
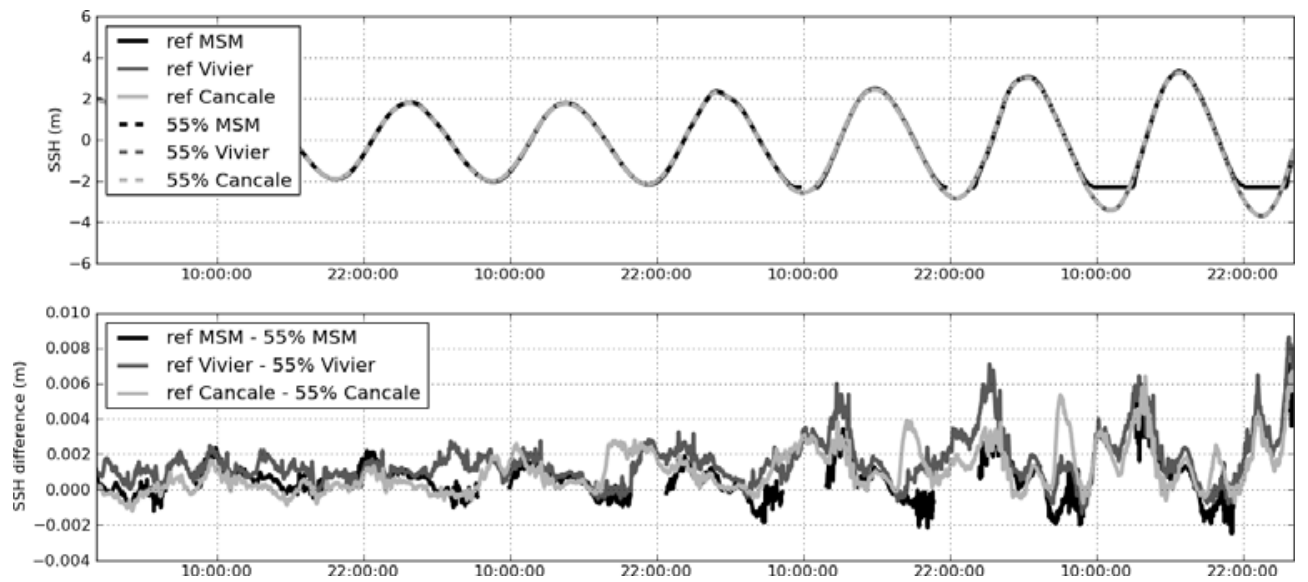

Figure 7. En haut : Élévation de la surface de la mer (SSH) aux points MSM, Vivier et

Cancale pour la simulation de référence sans hydrolienne et pour la simulation avec une extraction de 55\% de la puissance des courants disponible ; en bas : différences de niveaux aux points MSM, Vivier et Cancale entre la simulation de référence sans hydrolienne et la simulation avec une extraction de 55\%, du 11 au 14 février.
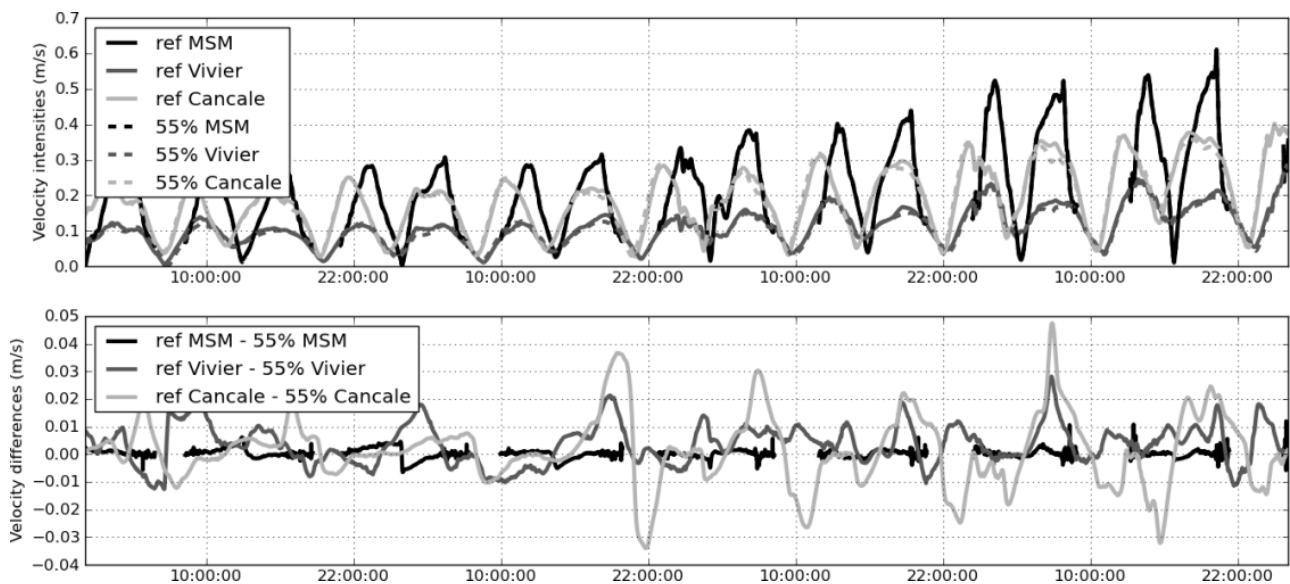

Figure 8. En haut : Intensité des vitesses aux points MSM, Vivier et Cancale pour la simulation de référence sans hydrolienne et pour la simulation avec une extraction de 55\% de la puissance des courants disponible ; en bas :différences d'intensité aux points

MSM, Vivier et Cancale entre la simulation de référence sans hydrolienne et la simulation avec une extraction de 55\%, du 11 au 14 février.

L'impact d'un parc d'hydroliennes sur les courants est ensuite évalué numériquement pour un parc situé dans cette zone. L'extraction d'énergie des courants est représentée par une force de retard ajoutée dans les équations du mouvement du code hydrodynamique MARS3D. Deux pourcentages d'extraction de l'énergie disponible sont présentés dans cette étude : $25 \%$ et 55\%. L'extraction d'énergie impacte fortement les courants localement mais également de manière plus faible et épisodique des zones plus éloignées. 
L'impact de la modification hydrodynamique sur la dynamique sédimentaire de la Baie est actuellement à l'étude. Les vagues seront également prises en compte dans ces simulations afin d'obtenir des remises en suspension de sédiments plus réalistes.

D’autre part, des études numériques et expérimentales sont également menées en collaboration avec le bassin d'essais hydrodynamiques Ifremer de Boulogne-sur-Mer afin de représenter plus finement les hydroliennes dans le modèle.

\section{Références}

AHMADIAN R., FALCONER R., BOCKELMANN-EVANS B. (2012). Far-field modelling of the hydro-environmental impact of tidal sream turbines. Renewable Energy, Vol. 38(1), pp 107-116. doi:10.1016/j.renene.2011.07.005

CAYOCCA F., DUSSAUZE M., LE HIR P., BASSOULLET P., JESTIN H. (2006). Modélisation hydro-sédimentaire de la Baie du Mont Saint-Michel. IXèmes JNGCGC, Brest, pp 157-166. doi:10.5150/jngcgc.2006.016-C

DEFNE Z., HAAS K.A., FRITZ H.M. (2011). Numerical modeling of tidal currents and the effects of power extraction on estuarine hydrodynamics along the Georgia coast, USA. Renewable Energy, Vol. 36(12), pp 3461-3471. doi:10.1016/j.renene.2011.05.027

DIVETT T., VENNELL R., STEVENS C. (2011). Optimisation of Multiple Turbine Arrays in a Channel with Tidally Reversing Flow by Numerical Modelling with Adaptive Mesh. In EWETEC Congress, Southampton, 2011.

EHROLD A. (1999). Dynamique de comblement d'un bassin sédimentaire soumis à un régime mégatidal : Exemple de la Baie du Mont Saint Michel. Thèse, Université de Caen, 294 p.

KERVELLA Y. (2010). Impact des installations ostréicoles sur l'hydrodynamique et la dynamique sédimentaire. Thèse, Université de Caen, 282 p.

LAZURE P., DUMAS F. (2008). An external-internal mode coupling for a 3D hydrodynamical model for applications at regional scale (MARS). Advances in Water Ressources, 31, pp 233-250. doi:10.1016/j.advwatres.2007.06.010

LE ROY R., SIMON B. (2003). Réalisation et validation d'un modèle de marée en Manche et dans le Golfe de Gascogne; Application à la réalisation d'un nouveau programme de réduction des sondages bathymétriques. Rapport d'études SHOM 002/03.

MYERS L.E., BAHAJ A.S. (2006). Power output performance characteristics of a horizontal axis marine current turbine, Renew. Energy, Vol. 31, pp.197-208. doi:10.1016/j.renene.2005.08.022

MYCEK P., GERMAIN G., PINON G., RIVOALEN E. (2011). Numerical and experimental study of interaction between two marine current turbines. In EWETEC Congress, Southampton, 2011. 